\title{
Estimating Constituency Preferences from Sparse Survey Data Using Auxiliary Geographic Information
}

\author{
Peter Selb \\ Department of Politics and Public Administration and \\ Center for Quantitative Methods and Survey Research, \\ University of Konstanz, PO Box D85, 78457 Konstanz, Germany \\ e-mail: peter.selb@uni-konstanz.de (correspondingauthor) \\ Simon Munzert \\ Center for Quantitative Methods and Survey Research, \\ University of Konstanz, PO Box D85, 78457 Konstanz, Germany \\ e-mail:simon.munzert@uni-konstanz.de
}

\begin{abstract}
Measures of constituency preferences are of vital importance for the study of political representation and other research areas. Yet, such measures are often difficult to obtain. Previous survey-based estimates frequently lack precision and coverage due to small samples, rely on questionable assumptions or require detailed auxiliary information about the constituencies' population characteristics. We propose an alternative Bayesian hierarchical approach that exploits minimal geographic information readily available from digitalized constituency maps. If at hand, social background data are easily integrated. To validate the method, we use national polls and district-level results from the 2009 German Bundestag election, an empirical case for which detailed structural information is missing.
\end{abstract}

\section{Introduction}

Constituency preferences, commonly conceptualized in terms of the distribution of the voters' issue attitudes or value orientations within electorally relevant geographical units, are central for studying classic questions about democratic representation. For example, does the roll call behavior of legislators reflect their constituents' policy views? Under what political and institutional conditions? What will be the electoral consequences if legislators fail to do so? Providing an empirical answer to such questions inevitably necessitates measures of constituency preferences. Yet, such measures are often difficult to obtain. Previous measures of constituency preferences based on aggregate proxies of public opinion such as constituency-level sociodemographics (e.g., Kalt and Zupan 1984; Krehbiel 1993; Levitt 1996), presidential electoral returns (e.g., Erikson and Wright 1980; Ansolabehere, Snyder, and Stewart 2001; Canes-Wrone, Brady, and Cogan 2002), and referenda outcomes (e.g., Kuklinski 1978; McCrone and Kuklinski 1979; McDonagh 1993) have been criticized both in validity and specificity terms (see Jackson and King 1989). Direct survey measures that average respondents' attitudes by district usually lack precision due to small samples and do not provide estimates for out-of-sample constituencies (e.g., Miller and Stokes 1963; McCrone and Stone 1986; Hurley and Hill 2003), or pool surveys over long time periods and therefore have to assume temporally stable preferences (e.g., Wright, Erikson, and McIver 1985; Gibson 1992; Erikson, Wright, and McIver 1993; Brace et al. 2002). Early model-based approaches that regress individual preferences on social background characteristics, and then use these parameter estimates to predict mean district preferences from the district-level distribution of covariates known from official 
sources (e.g., de Sola Pool, Abelson, and Popkin 1965; Weber et al. 1972; Sullivan and Minns 1976; Erikson 1978) are relatively insensitive to small samples and lack of district coverage. At the same time, however, such strategies involve cross-level inferences that rest on strong assumptions (see Seidman 1975). Ardoin and Garand (2003) take a top-down approach in estimating mean preferences in congressional districts by first regressing (large sample) state-level direct estimators of opinion on state-level characteristics. The first-stage regression weights are then used to predict mean district-level preferences from known population characteristics. Again, relationships between variables are not necessarily the same across spatial scales, a phenomenon that is similar to the ecological inference problem and known as the "modifiable areal unit problem" in the spatial statistics literature (see Openshaw and Taylor 1979).

Current hierarchical estimators of constituency preferences have been demonstrated to outperform previous approaches regarding their accuracy and efficiency (e.g., Park, Gelman, and Bafumi 2004) but require detailed structural information that will often be missing, for example, if the boundaries of the geographic units of interest (e.g., electoral districts) do not coincide with the boundaries of the administrative units for which this information is collected or provided.

In this paper, we propose an alternative Bayesian hierarchical estimation strategy that exploits auxiliary geographic information about the constituencies' neighborhood structure and surface area, which is readily available from digitalized constituency maps alone. If at hand, social background information is easily integrated in a manner compatible with other state-of-the-art strategies to estimating constituency preferences (Park, Gelman, and Bafumi 2004 in particular). In doing so, we draw on (and call attention to) recent developments in small area estimation (SAE) - a field that covers a variety of methods used to produce survey-based estimates for geographical areas in which the sample sizes are too small to provide reliable direct estimates (see Rao 2005), but has been largely ignored by scholars of representation. ${ }^{1}$

To validate the method, we estimate district-level party vote shares from two postelection surveys conducted after the 2009 German Bundestag election in order to compare them to official election results. Germany is a particularly relevant empirical case since detailed census data as required by alternative approaches are not available — partly for structural reasons, that is, nonnested administrative and electoral geographies, partly due to political sensibilities arguably dating back to the massive abuses of census data during the Nazi regime (see Luebke and Milton 1994). We also demonstrate how our approach may be combined with the methods of Park, Gelman, and Bafumi (2004), provided that demographic information for poststratification is available.

\section{The Current Gold Standard}

The major drawbacks of direct estimates of constituency preferences in normal circumstances, that is, their imprecision and potential lack of coverage with sparse survey data and large numbers of districts, arise from their exclusive reliance on survey information from within constituencies. ${ }^{2}$ Alternative strategies use supplementary cross-district information in various ways (for a recent review, see Berry et al. 2010). Among those, an approach by Gelman and Little (1997) and Park, Gelman, and Bafumi (2004) is currently emerging as a widely used gold standard for estimating constituency preferences from national surveys (e.g., Berkman and Plutzer 2005; Christensen and Florence 2008; Gelman 2008; Lax and Phillips 2009a; Hill, Herron, and Lewis 2010; Kastellec, Lax, and Phillips 2010).

Gelman and his colleagues pick up on the earlier idea of de Sola Pool, Abelson, and Popkin (1965) and Weber et al. (1972) to stratify survey samples into voter types, and to use census information for poststratification in order to obtain state-level constituency preferences. They develop a multilevel logistic model, with binary preferences $y$ of individuals $i=1,2, \ldots, N$ being modeled as a function of $S=64$ sociodemographic voter types $s$ as defined by ethnicity and sex (both binary), age and education (four

\footnotetext{
${ }^{1}$ The only explicit references to SAE that we came across in the vast political science literature on measuring constituency preferences appear in Jackson (1989) and Park, Gelman, and Bafumi (2004).

${ }^{2}$ For some notable exceptions using extraordinarily rich survey data that provide enough information to allow for accurate direct estimation, see Bafumi and Herron (2006), Clinton (2006), and Klingemann and Wessels (2001).
} 
categories $k$ and $l$ each) plus their respective interaction terms, nested in $J=51$ states $j$ (including Washington, DC), ${ }^{3}$

$$
\begin{aligned}
\operatorname{Pr}\left(y_{i}=1\right)= & \operatorname{logit}^{-1}\left(\beta^{0}+\beta^{\text {female }_{\text {female }}}+\beta^{\text {black }_{\text {black }_{i}}}\right. \\
& \left.+\beta^{\text {female.black }}(\text { female } \times \text { black })+\alpha_{k[i]}^{\text {age }}+\alpha_{l[i]}^{\text {edu }}+\alpha_{k[i],[i]}^{\text {age.edu }}+\alpha_{j[i]}^{\text {state }}\right),
\end{aligned}
$$

making for a total of $64 \times 51=3264$ synthetic subconstituencies. At first glance, stratification into a large number of subconstituencies thus seems to aggravate, not remedy, the problem of data scarcity. To compensate for small survey samples per (sub-)constituency, however, the hierarchical model "borrows strength" by partially pooling voter types across states

$$
\begin{gathered}
\alpha_{k}^{\text {age }} \sim \mathrm{N}\left(0, \sigma_{\text {age }}^{2}\right), \\
\alpha_{l}^{\text {edu }} \sim \mathrm{N}\left(0, \sigma_{\text {edu }}^{2}\right), \\
\alpha_{k, l}^{\text {age.edu }} \sim \mathrm{N}\left(0, \sigma_{\text {age.edu }}^{2}\right) .
\end{gathered}
$$

That is, all the respondents in a survey, no matter where they live, contribute information about differences in preferences between sociodemographic voter types to a degree warranted by the data. At the same time, the state of residency is used to estimate nondemographic state-level effects, which themselves are modeled using previous presidential vote shares as state-level predictors

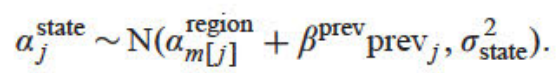

Also, regional effects are included to account for unobserved similarities among $M=5$ groups of states $m$ (Northeast, Midwest, South, West, and the District of Columbia)

$$
\alpha_{m}^{\text {region }} \sim \mathrm{N}\left(0, \sigma_{\text {region }}^{2}\right) .
$$

In a second step, Gelman and collaborators obtain measures of state-level preferences by summing over the predicted probabilities of holding the preference of interest $\pi$ for each stratification cell in proportion to their population share within each state

$$
\pi_{j}=\frac{\sum_{s \in j}^{S} \pi_{s} N_{s}}{N_{j}} .
$$

Park, Gelman, and Bafumi (2004) used the model to estimate state-level vote shares for George Bush, Sr. (therefore the logistic specification) at the 1988 and 1992 Presidential elections based on CBS NewsNew York Times polls with regularly sized national samples of 2193 and 4650 respondents. Validation by comparison to the actual election returns yields a mean absolute error of about $4 \%$ in both casesfar better than predictions based on separate state analyses (a strategy that is akin to the direct estimator based on small samples), and on complete pooling of all respondents across states (an approach similar to earlier two-step strategies based on standard regression methods). Lax and Phillips (2009a) further compared this method to the direct estimator based on a large-scale sample from several merged polls, and concluded that the hierarchical estimator using small samples is as accurate as utilizing 10 times as much survey data for direct estimation. Accordingly, the hierarchical estimator with poststratification appears to clearly outperform previous survey-based approaches in terms of its accuracy and efficiency. As opposed to the direct estimator, it offers coverage even for constituencies not included in the surveys used, provided constituency-level census information is available. Also, the hierarchical estimator incorporates the uncertainty inherent in direct estimation from survey data through shrinkage of directly observed differences between the constituencies contingent on the number of individual responses. Finally, by using census data for poststratification, this approach implicitly corrects for survey nonresponse, given that nonresponse is related to the observed sociodemographic characteristics.

${ }^{3}$ We lay out the model in some detail here to set the stage for a replication analysis to follow in Section 6 . 
On the other hand, though, the hierarchical estimator with poststratification requires detailed census information for the population of each synthetic voter type in each state (e.g., the number or population share of white females aged over 65 who are college graduates in Arizona)-information that might not be available for constituencies other than U.S. states or at lower federal levels. Likewise, whenever the aim is to estimate constituency preferences at lower federal levels, the number of stratification cells will rapidly increase. For example, a model including the above 64 sociodemographic categories and the 435 congressional districts will yield 27,840 synthetic subconstituencies. Although multilevel models borrow strength from across all observations, and thus do not require that each and every stratification cell be populated with survey respondents, such complexities will inevitably necessitate larger numbers of survey responses.

\section{An Alternative Approach Using Auxiliary Geographic Information}

So, what to do if the current gold standard in estimating constituency preferences is impractical due to data limitations? Our alternative approach starts from the observation that political predispositions, preferences, and behaviors often come along in geographic clusters (see, inter alia, Agnew 2002; Gelman 2008; Johnston and Pattie 2006). Explanations of these geographical clusterings emphasize regional differences in historical settlement and immigration patterns (Elazar 1994), the geography of industrialization and urbanization (Rodden 2010), and the resulting differences in current social composition (Hero 1998) and local economic conditions (Heppen 2003). Such broad historical processes are unlikely to abruptly halt at the geographical boundaries of constituencies, particularly if these boundaries are arbitrarily rather than historically drawn to satisfy numerical considerations. For example, single-member plurality (SMP) electoral districts are required to unite approximately equal numbers of voters to warrant evenly weighted votes and are thus often subject to redistricting in order to reflect changing shares of electoral population. If the geographical distribution of political preferences cross cuts constituency boundaries, this will lead to similar preference distributions among neighboring constituencies, that is, to spatial dependence. Potential spatial dependence is often conceived of as a nuisance for statistical estimation, as it may violate the assumption of independent errors that is typical of many regression models. Far from being treated as a nuisance, spatial dependence can be exploited to help solve sparse data problems of the sort described: provided constituency preferences are spatially correlated, the preferences of neighboring constituencies are informative about preferences in any particular constituency. Moreover, since most constituencies have more than one neighbor, the mean preferences in the neighborhood of a given constituency can usually be estimated more precisely than in the target area itself (subject to the condition that the number of survey respondents per constituency is relatively constant). One of the main issues is then to formally represent that spatial dependence in order to utilize it in the estimation problem.

To this end, we set up a - preliminarily empty - hierarchical model of the (logit of the) probability that a respondent $i=1,2, \ldots N$ in constituency $j=1,2, \ldots J$ holds a certain preference $y,{ }^{4}$

$$
\operatorname{Pr}\left(y_{i}=1\right)=\operatorname{logit}^{-1}\left(\alpha^{0}+\phi_{j[i]}+v_{j[i]}\right),
$$

where the constant term $\alpha^{0}$ represents a global propensity to hold that preference, and $\phi_{j}$ and $v_{j}$ both represent constituency-specific deviations from that global propensity which are assumed to be independent of each other. In particular, $\phi_{j}$ depicts a spatially correlated random effect (RE) for which we adopt an intrinsic conditional autoregressive (CAR) distribution (see Besag, York, and Molli 1991). ${ }^{5}$ Under this

\footnotetext{
${ }^{4}$ We use a logistic specification since, in the following empirical section, we will estimate party vote shares from poselection surveys in order to compare them to official election results (also see Park, Gelman, and Bafumi 2004). One could equally start from a linear model specification if indicated.

${ }^{5}$ Spatially correlated REs have relatively rarely been capitalized on in SAE until recently (see Pfefferman 2002). As a simple alternative to this specification, one could assume a simultaneous autoregressive (SAR) process where the RE of any two constituencies are spatially correlated as a smooth function of their distance (see Pratesi and Salvati 2008). However, we prefer the intrinsic CAR specification, as SAR processes involve assumptions (e.g., spatial stationarity) that are frequently violated in social science applications (see Cressie 1993).
} 
specification, the conditional distribution of $\phi_{j}$ given the $\phi$ 's in all the other constituencies $k \neq j$ depends only on the $\phi$ 's in the constituencies adjacent to $j$,

$$
\phi_{j} \mid \phi_{k} \sim \mathrm{N}\left(\frac{\sum_{k \neq j} w_{j k} \phi_{k}}{\sum_{k \neq j} w_{j k}}, \frac{\sigma_{\phi}^{2}}{\sum_{k \neq j} w_{j k}}\right),
$$

where the $w_{j k}$ are elements of a $J \times J$ adjacency matrix assuming a value of 1 if units $j$ and $k$ are neighbors, that is, have a common border or vertex, otherwise 0 . Hence, the expected conditional mean of $\phi$ in $j$ corresponds to the average value of $\phi$ in the neighborhood of $j$, with its variance parameter, $\sigma_{\phi}^{2}$, controlling how similar $\phi_{j}$ is to its neighbors. Deviation $v_{j}$, on the other hand, is assumed to vary independently and identically across districts according to a normal distribution,

$$
v_{j} \sim \mathrm{N}\left(0, \sigma_{v}^{2}\right) .
$$

Including both a spatially structured and an independent random component into the model will, in effect, pull the directly (but, due to small $N_{j}$, inaccurately) observed proportion of respondents holding the preference in constituency $j$ toward both its neighborhood and the overall sample mean, with the amount of shrinkage increasing with decreasing $N_{j}$. That is, inferences for the district-level parameters, $\pi_{j}=\operatorname{logit}^{-1}\left(\alpha^{0}+\phi_{j}+v_{j}\right)$, reflect not just the direct survey information in district $j$, but also draw on relevant information in the neighboring districts (which will normally host more respondents than $j$ ), as well as in all the other districts (i.e., the whole survey sample). The relative amount of local versus global smoothing is then determined by the estimated variance $\sigma_{\phi}^{2}$ in proportion to $\sigma_{v}^{2}$. Further, by exploiting the conditional distribution of $\phi_{j}$, the model equally informs estimates of constituency preferences for areas not covered by the survey, provided a constituency is not an island (i.e., it has neighbors to draw information from). ${ }^{6}$

If available, the model can easily be extended to include covariate information. In the following empirical application, we will use a single constituency-level covariate, namely the log inverse surface area, as a proxy of urbanity, ${ }^{7}$ which has been repeatedly demonstrated to be a crucial contextual determinant of political preferences, and therefore has the potential to disrupt otherwise smooth spatial preference distributions (see Rodden 2010). To this end, we move the constant term $\alpha^{0}$ from the individual-level model,

$$
\operatorname{Pr}\left(y_{i}=1\right)=\operatorname{logit}^{-1}\left(\alpha_{j[i]}+\phi_{j[i]}\right),
$$

to the district-level model, which now also includes the covariate

$$
\alpha_{j}=\alpha^{0}+\beta^{\text {area }} \log \left(\operatorname{area}_{j}^{-1}\right)+v_{j} .
$$

To better be able to separate between eventual improvements in predictive power due to the inclusion of the covariate and due to the spatially structured RE, we will pursue a sequential model strategy. First, we will fit an empty model containing only the unstructured RE, $v_{j}$ (Model 1), followed by a model including inverse area as a district-level covariate plus the unstructured RE (Model 2). Finally, we will add the spatially structured RE, $\phi_{j}$, and fit the full specification given in equations (9) and (10) (Model 3). Validation results will also be reported for the direct estimator of the constituency proportions of respondents holding the preference of interest (Direct), that is, $\frac{\sum_{i \in j}^{N_{j}} y_{i}}{N_{j}} .8$

\footnotetext{
Gote that, by exploiting second- and higher-order neighborhood relations, this is also true for constituencies that altogether lack in-sample neighbors, that is, all its neighbors have missing values as well. However, inferences will be imprecise and unstable in these circumstances.

7If (and only if) the number of residents is relatively constant across electoral districts, as is usually the case in SMP systems, surface area is logically related to population density. In situations where some structural information is available one could, of course, directly use population density as an indicator of urbanity. In fact, information about population density is available in our empirical case, and log inverse area and log population density are correlated at 0.99 . For the time being, we would like to see how far we get using minimal geographic information that is available from digitalized maps alone.

8The direct estimators may be weighted inversely proportional to the respondents' selection probability as determined by the sampling design of the survey. However, integrating such weights into a hierarchical model is not a trivial exercise (see Gelman 2007).
} 


\subsection{Estimation}

Given the paucity of survey and auxiliary data one usually confronts, the REs $\phi_{j}$ and $v_{j}$ are essential components of our model. However, the model is clearly overparameterized by including two REs per constituency, so the likelihood will only identify them jointly for each unit. ${ }^{9}$ The nature of the prior distributions in equations (7) and (8) allows posterior identifiability within a fully Bayesian setting, given the constraint that $\sum_{j=1}^{J} \phi_{j}=0$ (see Carlin and Louis 1996). Inference proceeds via Markov chain Monte Carlo (MCMC) methods to compute a joint posterior density for all the model parameters. We use WinBUGS to run the required computations (Lunn et al. 2000). Complex covariance structures as those in equation (7) can be implemented using GeoBUGS, an add-on module to WinBUGS for manipulating spatial data (Thomas et al. 2004). Commented replication code is given in the Appendix.

We use uninformative priors to let the data determine the parameters. In particular, we use flat priors for the fixed parameters, $\alpha^{0}$ and the $\beta$ 's, and vague uniform priors within a range of $(0,2)$ for the standard deviations of the REs, $\sigma_{\phi}$ and $\sigma_{v} \cdot{ }^{10}$ To monitor convergence, we set up three chains with randomly chosen starting values for the parameters, each with 20,000 iterations, of which we discard the first 10,000 before summarizing the parameters' posterior probabilities.

\subsection{Validation}

To validate our models, we estimate district-level party vote shares from two postelection surveys conducted after the 2009 German Bundestag election within the framework of the German Longitudinal Election Study (GLES) and compare them to their true distributions which are known from official election results. ${ }^{11}$ Germany's so-called "mixed-member proportional representation" electoral system divides the country into $J=299$ primary electoral districts (Wahlkreise), which constitute our target areas. The districts are nested within a higher electoral tier, the 16 Länder (federal states). Each voter has two votes: a candidate vote (Erststimme), which governs the allocation of the district seats (Direktmandate) using SMP, and a second vote (Zweitstimme) for a länder-specific party list that translates into seats (Listenmandate) according to the Sainte-Laguë method, restricted by a national 5\% clause (for details, see Saalfeld 2008). Since the Erststimme is particularly prone to strategic voting which may disrupt otherwise smooth spatial distributions of (partisan) preferences contingent on district-specific tactical incentives, we will focus our analysis on the Zweitstimme. All five parliamentary parties will be considered: the Christian Democrats (CDU/CSU), the Social Democrats (SPD), the Liberals (FDP), the Greens (B90/Die Grünen), and the Left (Die Linke). Altogether, these parties received $94 \%$ of the Zweitstimmen in 2009, which almost makes for the complete choice set with which the voters were confronted. Instead of adding a lumping category for marginal parties and setting up a more sensitive multinomial logistic regression that accommodates the fact that district-level party vote shares sum to unity, we model the party vote shares separately: first, in order to better be able to explore the party-specific conditions of how well or poorly the estimates perform; and second, to be consistent with the more general modeling strategy proposed in the previous section. Although estimating (multi-)party vote shares from survey data allows us to validate our method using real data, we suspect that there are not too many substantively interesting applications that deal with such compositional data. ${ }^{12}$

In validation terms, one important criterion will be the distance between true values and their estimates expressed as the mean absolute error (MAE) of (the median of) the estimates' posterior probabilities. Clearly, smaller MAEs indicate better point estimates. As to these estimates' uncertainty, we calculate $90 \%$ Bayesian credible intervals from the highest posterior density regions that can be immediately interpreted in terms of the probability that the true value of the estimated parameter is inside a given

\footnotetext{
${ }^{9}$ Additionally, due to its independent distribution, $v_{j}$ cannot be identified at all for constituencies that are not covered by the survey and thus has to be set to zero in these instances.

${ }^{10}$ Although results should be relatively insensitive to prior choice with a large number of districts (see below), we have also used alternative inverse gamma priors for the variances, $\sigma_{\phi}^{2}$ and $\sigma_{v}^{2}$, yielding almost identical point estimates (though marginally lower coverage probabilities).

${ }^{11}$ Official election statistics, digitalized maps, and some additional structural information can be downloaded from the web site of the Bundeswahlleiter (federal elections officer) at http://www.bundeswahlleiter.de/de/bundestagswahlen/BTW_BUND_09/.

${ }^{12} \mathrm{See} \mathrm{Katz}$ and King (1999) for a discussion of the potential issues with the separate modeling of multinomial proportions.
} 

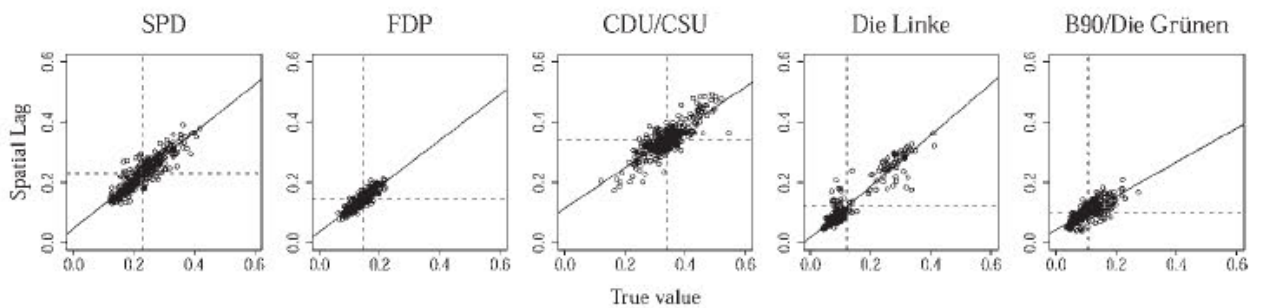

Fig. 1 Moran's plots: (true) district-level party vote shares versus their spatial lags, that is, the average party vote shares in the districts' neighborhoods. Official results from the German Bundestag election 2009.

interval. ${ }^{13} \mathrm{~A}$ second validation criterion is then the coverage probability of these credible intervals, that is, the proportion of the time that the intervals actually contain the true value of interest. The actual coverage probability should approximate the nominal level of $90 \%$ as closely as possible. A final validation criterion will be the estimates' efficiency in terms of the width of the credible intervals. With the coverage probability being equal, a narrower interval is, of course, preferable.

In addition to these validation criteria, we will also use a diagnostic tool to assess our models' assumption that RE $v_{j}$ is, indeed, independently distributed across districts. To this end, we employ Moran's $I$, a widely used measure of spatial dependence. Moran's $I$ indicates, in this case, the correlation between $v_{j}$ with its spatial lag, $\frac{\sum_{k \neq j}^{J} w_{j k} v_{k}}{\sum_{k \neq j}^{J} w_{j k}}$, the latter of which gives the average value of $v$ in the neighborhood of unit $j$ (see the definition of $w_{j k}$ following equation (7)).

\subsection{An Initial Check of the Model's Central Proposition}

The Moran statistic can equally be utilized for an initial check of our modeling approach's basic premise that political preferences often come along in geographical clusters. Figure 1 plots the official vote shares of the five parties (henceforth "true values") against their spatial lags. The slopes of the solid lines is given by the Moran values. The dashed lines represent averages of the true values and their spatial lags. The average number of neighbors per district is 5.5, with a minimum value of 1 and a maximum of 10 . Altogether, the magnitude of spatial autocorrelation is considerable, indicating that neighborhoods should be highly informative for estimating constituency preferences in any given district. In particular, the SPD vote shares exhibit the strongest spatial autocorrelation $(I=0.79)$, followed by the FDP $(0.76)$ and the $\mathrm{CDU} / \mathrm{CSU}(0.67)$. Although at first glance, there also seems to be tremendous autocorrelation in Die Linke vote shares ( 0.85 ), this turns out to be an artifact of its pronounced regional stronghold in eastern Germany (see the upper cloud in the graph). Separate calculations of Moran's $I$ yield lower yet substantial values of 0.57 for eastern and 0.49 for western districts. The same holds for B90/Die Grünen, whose district-level vote shares are spatially autocorrelated at 0.56 . Such variability across parties will further provide us with the opportunity to see how our estimates' performance depends on the true spatial correlation of partisan preferences.

\section{Survey Data}

Clearly, estimating constituency preferences for the 299 electoral districts from national polls puts some demands on the survey data even if model-based (let alone direct) estimation strategies are employed. We can benefit from the fortunate circumstance that the 2009 GLES conducted two surveys after the election on September 27:14 a face-to-face (F2F) survey of a three-stage random sample of 2117 eligible

\footnotetext{
${ }^{13}$ Assessing the uncertainty of the direct estimators via (frequentist) confidence intervals is far from straightforward with small samples (see Agresti and Coull 1998). Moreover, frequentist and Bayesian intervals are hardly comparable, as the former treat the estimated parameters as fixed and the confidence intervals as random, so that the probability that the true parameter value is inside the given interval is either 0 or 1 . In the subsequent empirical analysis, we will therefore only report MAEs for the direct estimators but no uncertainty assessments.

${ }^{14}$ The survey data as well as technical reports are available from the GLES Web site (www.gesis.org/gles).
} 
Table 1 Summary statistics of the survey data utilized

\begin{tabular}{lcccccc}
\hline & $N$ & $J$ & $\bar{N}_{j}$ & $S D\left(N_{j}\right)$ & $\min \left(N_{j}\right)$ & $\max \left(N_{j}\right)$ \\
\hline F2F & 1527 & 201 & 7.60 & 5.21 & 1 & 29 \\
CATI & 3450 & 291 & 12.16 & 5.60 & 2 & 45 \\
Pooled & 5067 & 297 & 17.06 & 8.31 & 1 & 51 \\
\hline
\end{tabular}

Note. $N$, numbers of respondents; $J$, numbers of districts covered; $\bar{N}_{j}$, average numbers of respondents per district, their standard deviations, minimum and maximum values; F2F, face-to-face postelection survey; CATI, postelection wave of a computer-aided telephone rolling cross-sectional survey; pooled, F2F and CATI surveys pooled.

individuals, and the postelection wave of a rolling cross-section survey of 4027 individuals conducted by telephone (computer-assisted telephone interviewing [CATI]) ${ }^{15}$ For the time being, we will pool these data, which makes for a total sample of 6144 respondents, 5067 of which are self-reported voters that indicated a party choice (recall that our aim is to estimate party vote shares, which are fractions of actual voters). Subsequently, we will also use the two surveys separately in order to see how our estimates behave with more common sample sizes. Table 1 gives an overview over the sample characteristics. The pooled sample covers 297 of the 299 districts, with an average of $\bar{N}_{j}=17$ respondents per district, ranging from 1 to 51 - barely sufficient to directly estimate the vote shares of the five parties in any remotely reliable manner. Of course, this holds all the more true for the separate surveys. Otherwise, the global sample vote shares of the parties quite reasonably reflect their true national-level values, with B90/Die Grünen being the most notable exception ( $13.7 \%$ according to the pooled sample versus an official return of $10.7 \%$ ) -an issue we will return to in Section 6.

\section{Results}

Figure 2 plots the true party vote shares versus the direct estimates and the medians of the model-based estimates' posterior densities. Above all, it is easy to see that the direct estimates graphed in the leftmost panels are essentially useless in all cases. They are way too variable and wide off the mark in most instances - in fact, so wide off the mark that some of them could not even be plotted within the displayed range of $0-60 \%$ of the votes. As the MAEs reported in Table 2 show, they misestimate the true vote shares by an unsatisfactory $7.4-10.1 \%$. The estimates from the unstructured hierarchical model in the second column (Model 1) generally go to the other extreme and shrink the direct estimates toward the overall sample means (represented by the dashed lines in Fig. 2) to an extent that there is barely any cross-district variability left. Adding log inverse area as a proxy of population density/urbanity to the unstructured RE model in the third column (Model 2) improves the estimates of the CDU/CSU, B90/Die Grünen, and very modestly, the SPD vote shares. ${ }^{16} \mathrm{~A}$ more considerable improvement of the estimates results from adding the spatially structured RE, $\phi_{j}$, in the final column (Model 3). The models' point estimates come out very close to their true values, with an average absolute error ranging from just $2.6 \%$ for the FDP to $4.5 \%$ for the CDU/CSU. The CDU/CSU estimates are too conservative in the tails, which also leads to coverage probabilities relatively far below their nominal level. Nevertheless, the coverage probabilities of the credible intervals of the spatially informed estimates are generally much closer to their nominal $90 \%$ level than those of the previous estimators; meanwhile, the intervals are narrower and thus more efficient. Finally, as indicated by the Moran's values in Table 2, the inclusion of $\phi_{j}$ has helped to rid most of the

\footnotetext{
${ }^{15}$ The respondents from the F2F survey were sampled using the standard design of the consortium of German market research institutes (Arbeitsgemeinschaft deutscher Marktforschungsinstitute, ADM), with electoral wards (Wahlbezirke) as the primary sampling units (PSUs) being randomly sampled within regions (East and West), households being selected through random-route methods within electoral wards, and persons being randomly chosen within households. The sample of the CATI survey was drawn using the so-called Gabler-Häder design, where area codes within regions serve as the PSUs. None of these designs will normally warrant equal probability samples for the electoral districts. Absent the information necessary to construct weights that compensate for unequal selection probabilities within districts (and the quibbles with including such weights in hierarchical models; see Footnote 8), we will treat the district samples as if they were randomly drawn.

${ }^{16} \mathrm{CDU} / \mathrm{CSU}$ vote shares are negatively, and SPD and B90/Die Grünen vote shares positively related to urbanity (see the estimated coefficient plot in Fig. 3). FDP and Die Linke vote shares are also related to urbanity, although in a nonmonotonic way that is difficult to interpret, let alone predict, theoretically.
} 

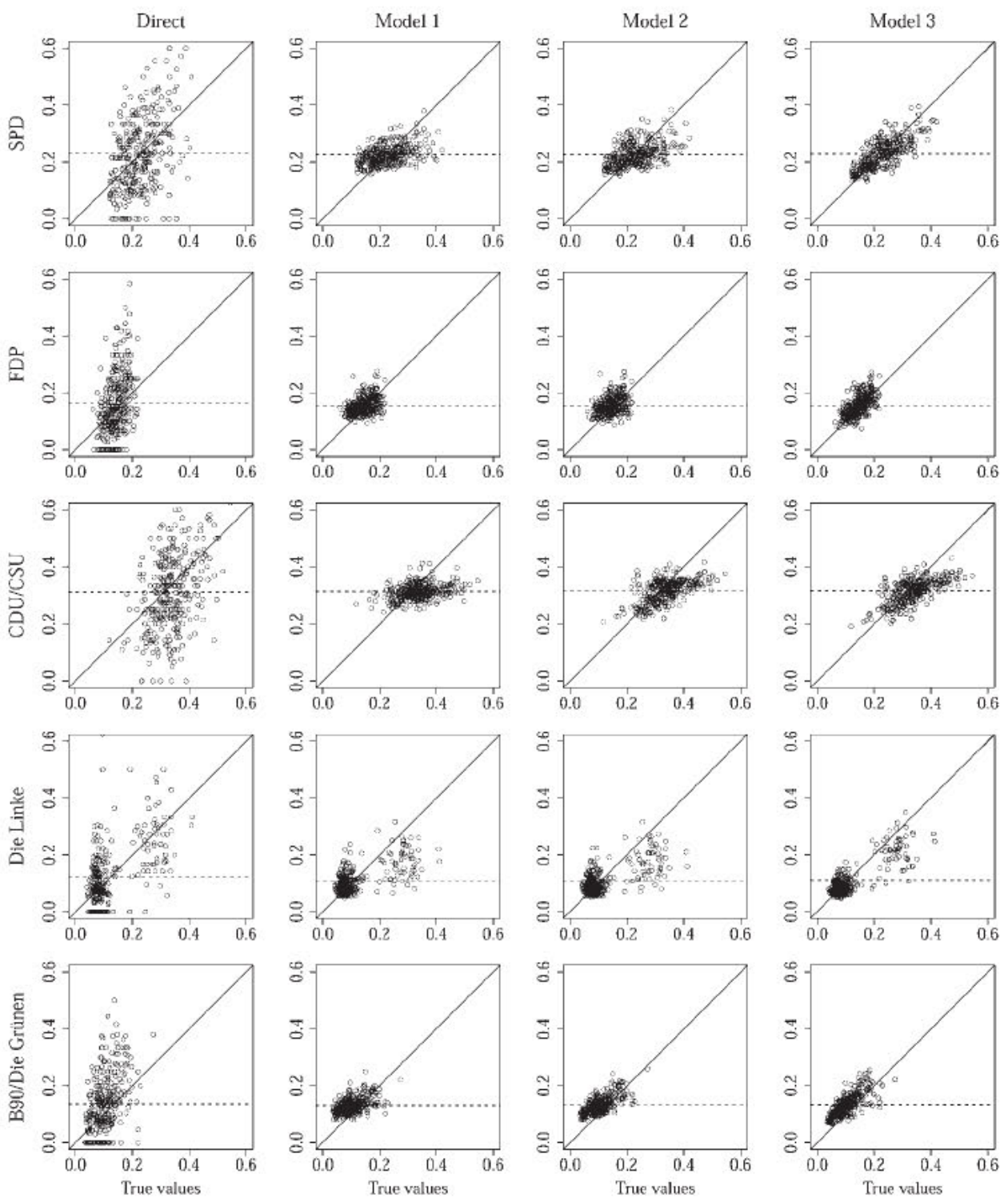

Fig. 2 True district-level party vote shares versus estimates from alternative model specifications: direct estimates (Direct), estimates from an empty hierarchical model including an unstructured RE (Model 1), from a model including $\log$ inverse area as a covariate and an unstructured RE (Model 2), and from a model including the district-level covariate and both unstructured and spatially structured REs (Model 3).

spatial autocorrelation of $v_{j}$ (which is assumed i.i.d.) in the previous models, ${ }^{17}$ which supports the view that the CAR-structured REs provide a good representation of the prevalent spatial patterns. The only notable exception in this regard is the CDU/CSU model. Overall, our results suggest that the estimates from Model 3 perform remarkably well with the pooled data, particularly considering the models' exclusive reliance on auxiliary geographic information. The estimators' performance seemingly depends on the amount of spatial autocorrelation inherent in the underlying distributions of the true preferences. In particular, the model performed better in estimating SPD and FDP vote shares and worse in estimating the

\footnotetext{
${ }^{17}$ Another violation obvious from Fig. 2, the assumption that $v_{j}$ is normally distributed, also seems difficult to maintain for the model of Left vote shares. Rather, there seem to be distinct modes for eastern and western districts. We therefore added a regional dummy to the vector of covariates that reduced the Left model's MAE to 0.02 , although at the expense of overconfident estimates, as indicated by a decline in mean coverage probabilities to .83 . Detailed results are available upon request.
} 
Table 2 Validation results for the estimates presented in Fig. 2

\begin{tabular}{|c|c|c|c|c|c|c|c|c|c|c|c|c|c|}
\hline & \multicolumn{4}{|c|}{ Mean absolute error } & \multicolumn{3}{|c|}{ Mean width of $90 \% \mathrm{CI}$} & \multicolumn{3}{|c|}{ Coverage probabilities } & \multicolumn{3}{|c|}{ Moran's I } \\
\hline & Direct & Model 1 & Model 2 & Model 3 & Model 1 & Model 2 & Model 3 & Model 1 & Model 2 & Model 3 & Model 1 & Model 2 & Model 3 \\
\hline \multicolumn{14}{|l|}{ Pooled, $N=5067$} \\
\hline SPD & 0.096 & 0.047 & 0.047 & 0.034 & 0.181 & 0.165 & 0.141 & .893 & .846 & .920 & $0.062 *$ & $0.063^{*}$ & -0.037 \\
\hline FDP & 0.079 & 0.029 & 0.030 & 0.026 & 0.157 & 0.158 & 0.145 & .946 & .943 & .963 & 0.011 & 0.014 & -0.060 \\
\hline $\mathrm{CDU} / \mathrm{CSU}$ & 0.101 & 0.052 & 0.048 & 0.045 & 0.178 & 0.159 & 0.156 & 816 & .836 & .846 & $0.110^{*}$ & $0.106^{*}$ & $0.073^{*}$ \\
\hline Die Linke & 0.074 & 0.044 & 0.044 & 0.032 & 0.168 & 0.168 & 0.126 & .886 & .890 & .906 & $0.273^{*}$ & $0.248^{*}$ & 0.032 \\
\hline B90/Die Grünen & 0.075 & 0.037 & 0.033 & 0.033 & 0.146 & 0.132 & 0.123 & .783 & .786 & .796 & -0.014 & -0.007 & -0.130 \\
\hline \multicolumn{14}{|l|}{ F2F, $N=1527$} \\
\hline SPD & 0.101 & 0.052 & 0.051 & 0.043 & 0.160 & 0.158 & 0.212 & .702 & .722 & .953 & 0.085 & 0.095 & 0.016 \\
\hline FDP & 0.100 & 0.032 & 0.033 & 0.037 & 0.140 & 0.146 & 0.204 & .796 & .816 & .970 & 0.058 & 0.051 & -0.000 \\
\hline $\mathrm{CDU} / \mathrm{CSU}$ & 0.119 & 0.063 & 0.063 & 0.067 & 0.220 & 0.229 & 0.284 & .729 & .753 & .910 & $0.218^{*}$ & $0.212^{*}$ & $0.162 *$ \\
\hline Die Linke & 0.093 & 0.064 & 0.064 & 0.054 & 0.175 & 0.174 & 0.211 & .605 & .619 & .876 & $0.177^{*}$ & $0.184^{*}$ & 0.115 \\
\hline B90/Die Grünen & 0.076 & 0.032 & 0.029 & 0.033 & 0.101 & 0.107 & 0.146 & .742 & .793 & .930 & $0.324^{*}$ & $0.306^{*}$ & $0.297^{*}$ \\
\hline \multicolumn{14}{|l|}{ CATI, $N=3540$} \\
\hline SPD & 0.107 & 0.051 & 0.051 & 0.043 & 0.136 & 0.123 & 0.135 & .726 & .635 & .793 & 0.043 & 0.035 & -0.017 \\
\hline FDP & 0.089 & 0.030 & 0.032 & 0.030 & 0.106 & 0.113 & 0.122 & .783 & .793 & .880 & 0.025 & 0.038 & 0.007 \\
\hline $\mathrm{CDU} / \mathrm{CSU}$ & 0.114 & 0.058 & 0.052 & 0.049 & 0.123 & 0.108 & 0.133 & .619 & .615 & .759 & 0.028 & 0.038 & 0.004 \\
\hline Die Linke & 0.078 & 0.045 & 0.046 & 0.033 & 0.164 & 0.165 & 0.124 & .876 & .880 & .930 & $0.283^{*}$ & $0.281^{*}$ & 0.063 \\
\hline B90/Die Grünen & 0.085 & 0.048 & 0.044 & 0.043 & 0.130 & 0.103 & 0.120 & .639 & .538 & .649 & 0.036 & $0.085^{*}$ & -0.053 \\
\hline
\end{tabular}

Note. CATI, postelection wave of a computer-aided telephone rolling cross-sectional survey; pooled, F2F and CATI surveys pooled.

${ }^{*} p<.10$ (for Moran values). 
vote shares of the other parties, most notably those of B90/Die Grünen, with an unacceptably low mean coverage probability of .76. A closer inspection of Fig. 2 reveals, though, that the modest performance of the estimates of the Green vote shares is primarily due to the general upward bias in reported votes that has already been remarked in the data section above. We will further discuss this issue in the following section.

Finally, how well do the estimators perform with more regular sample sizes, that is, the F2F ( $N=1527)$ and the CATI $(N=3540)$ surveys taken separately? Not surprisingly, the MAEs are generally larger than those for the pooled data, ranging at best (again with the spatially informed estimators) from $3.3 \%$ to $6.7 \%$ in the former, and from $3.0 \%$ to $4.9 \%$ in the latter case (see the two lower panels in Table 2). It is difficult to tell at first sight whether these differences are due to the CATI survey providing larger $N$ (and thus, larger $\bar{N}_{j}$ ), or fuller cross-district coverage in terms of $J$ (Table 1), or due to the possibility that a telephone survey sample more evenly represents the district populations than the F2F sample based on single electoral wards within districts (see Footnote 15). On closer inspection (see the additional plots in the online Appendix), the MAEs of the spatially informed estimates generally proved relatively insensitive to the number of respondents per district, $N_{j}$. Furthermore, even the estimates for the off-sample districts (i.e., $N_{j}=0$ ) on average did not come out wider off target than those for the in-sample districts. Rather, the accuracy of the Model 3 estimates seem to moderately depend on the number of respondents in the neighborhood of a given district. Curiously, though, the estimators based on the CATI survey are overconfident and thus exhibit lower coverage probabilities than those based on the F2F survey. In general, these results indicate that, for obtaining accurate model-based estimates for so many small areas, one still necessitates relatively large-scale national polls.

\section{Combining Both Approaches}

One of the drawbacks of our approach is that it does not naturally correct for survey nonresponse. This problem has, perhaps, become most evident in the general upward shift of our district estimates of Green party vote shares. In this final part of our empirical analysis, we will therefore demonstrate how our approach may be beneficially combined with the method of Park, Gelman, and Bafumi (2004) using poststratification to population controls in order to correct for nonresponse bias in this case. Subsequently, we will turn the tables and replicate the original analysis of Park, Gelman, and Bafumi (2004) to see whether our neighborhood-based estimation strategy offers added value even in situations where sociostructural information abounds.

As to the presumed nonresponse problem, it is well known that both the individual propensity to support postmaterialist parties and to participate in election surveys is positively related to educational attainment (e.g., Huefken 2010). If so, we would expect the higher educated to be overrepresented in our sample, and the upward bias in our estimates of Green party vote shares to decrease after poststratification to the "true" district-level distributions of attainment levels. Although census data on education are not available, the Bundeswahlleiter at least provides district-level school graduation statistics as of 2007 which we, in the absence of better alternatives, use as proxies for the whole district populations (see Footnote 14). Indeed, individuals with tertiary education seem to be massively overrepresented in our sample ( $42 \%$ compared to $27 \%$ according to the official statistic). In contrast, those with secondary ( $33 \%$ compared with $41 \%$ ) and primary education ( $24 \%$ compared with $32 \%$ ) are underrepresented. ${ }^{18}$ Therefore, we rerun our models of the Green party vote shares adding dummy variables for the two higher levels of educational attainment to the individual-level model in equation (9) (see the bottom panel of Fig. 3 for the estimates), ${ }^{19}$

$$
\operatorname{Pr}\left(y_{i}=1\right)=\operatorname{logit}^{-1}\left(\alpha_{j[i]}+\beta^{\text {edu2 }} \text { edu } 2_{i}+\beta^{\text {edu } 3} \operatorname{edu} 3_{i}+\phi_{j[i]}\right),
$$

and obtain predicted probabilities of having cast a Green vote for each of the $299 \times 3=897$ crossclassifications of electoral districts and attainment levels. The district estimate is then simply the population-weighted average of the attainment-specific predicted probabilities by district (see equation (5)).

\footnotetext{
${ }^{18}$ Considering that younger cohorts as those having graduated in 2007 are, on average, higher educated than older cohorts, we may even suspect the true educational bias in our sample to be stronger than indicated.

${ }^{19}$ As opposed to Park, Gelman, and Bafumi (2004) in equations (3) above, we model education with fixed, not REs.
} 

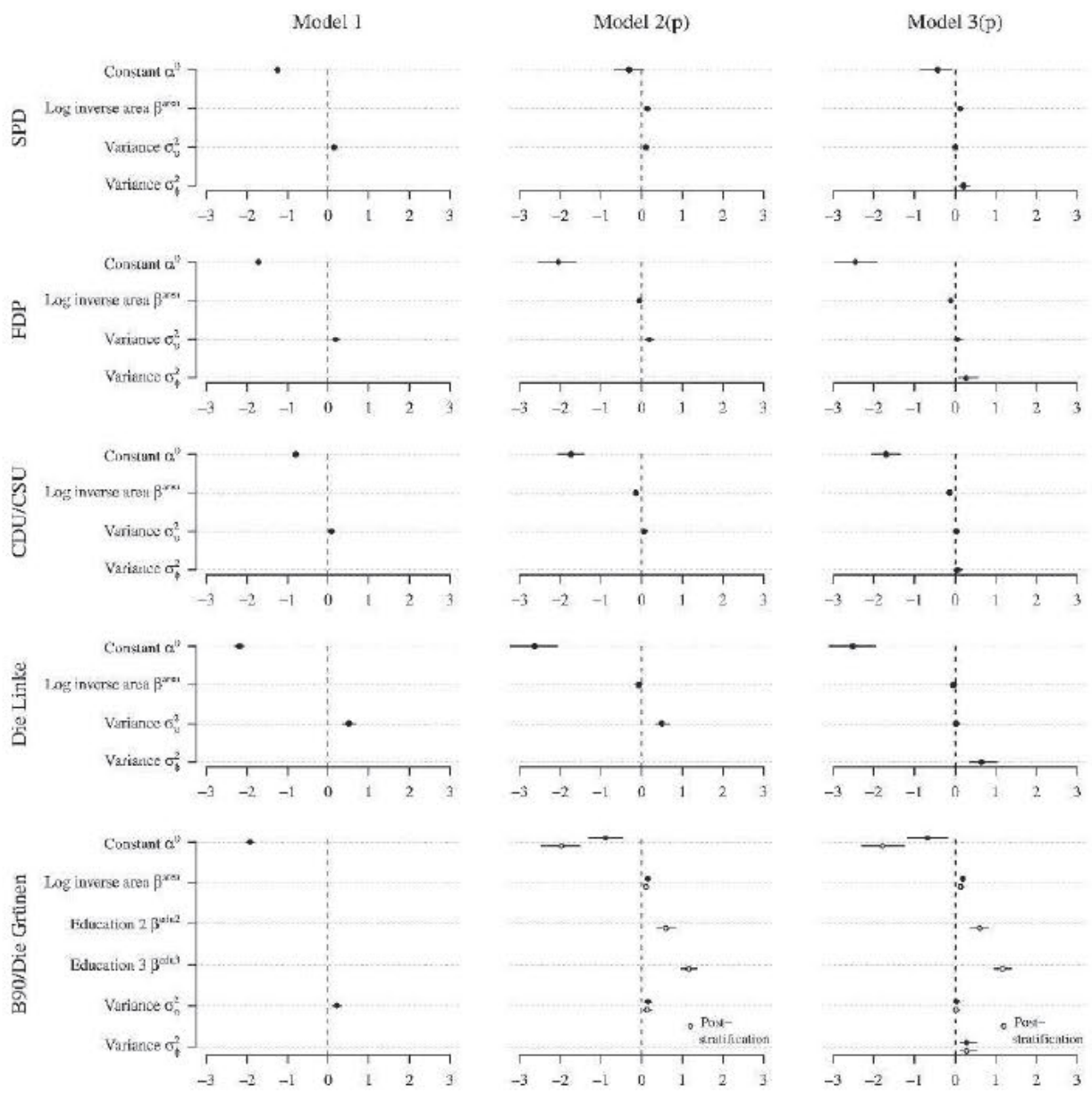

Fig. 3 Medians and 90\%-credible intervals of the coefficients' Bayesian posterior probabilities from different specifications of the hierarchical model of the respondents' partisan preferences: An empty model including an unstructured RE (Model 1), a model including log inverse area as a covariate and an unstructured RE (Model 2), and a model including the district-level covariate and both unstructured and geographically structured REs (Model 3). Green party vote shares have been additionally modeled including higher educational attainment levels for poststratification to district population controls (Models $2 \mathrm{p}$ and $3 \mathrm{p}$ ).

The results for the estimates from a model including only the unstructured RE in addition to the covariates (Model 2p), and both unstructured and CAR-structured REs (Model 3p) are reported in Table 3. Obviously, the upward bias in the estimates observed in the previous section is considerably reduced by using poststratification. Both MAEs are markedly lower (just $2.8 \%$ and $2.6 \%$, respectively), and the coverage probabilities are more or less exactly at their nominal level of $90 \%$ for both estimators. Also, the inclusion of educational attainment seems to have soaked up most of the spatial autocorrelation previously present in $v_{j}$, as indicated by the insignificant value of the Moran statistic. But still, the spatially informed estimator is slightly more precise in terms of MAE and more efficient in terms of a narrower credible interval.

Having demonstrated how poststratification information can be fruitfully integrated into our model using the method of Park et al., we now turn to original analysis of a 1988 election poll of Park, Gelman, and Bafumi (2004) to see what our neighborhood-based estimation strategy has to offer in reverse. ${ }^{20}$

\footnotetext{
20Replication data and code for this analysis is available from Andrew Gelman's Web site (http://www.stat.columbia.edu/ gelman/ arm/examples/election88/). We have used a digitalized states map from Kenneth Foote's Web site (http://www.colorado.edu/ geography/foote/maps/assign/hotspots/hotspots.html).
} 
Table 3 Validation results for the hierarchical estimators of Green party vote shares with poststratification to district-level educational attainment. Specifications with an unstructured RE (Model 2p) and both unstructured and spatially structured REs (Model 3p)

\begin{tabular}{|c|c|c|c|c|c|c|c|}
\hline \multicolumn{2}{|c|}{ Mean absolute error } & \multicolumn{2}{|c|}{ Mean width of $90 \% C I$} & \multicolumn{2}{|c|}{ Coverage probabilities } & \multicolumn{2}{|c|}{ Moran's I } \\
\hline Model $2 p$ & Model $3 p$ & Model $2 p$ & Model $3 p$ & Model $2 p$ & Model $3 p$ & Model $2 p$ & Model $3 p$ \\
\hline 0.028 & 0.026 & 0.143 & 0.139 & .886 & .916 & 0.001 & -0.134 \\
\hline
\end{tabular}

${ }^{*} p<.10$ (for Moran values).

As depicted in equation (4), the model of Park et al. includes a regional RE and thus already provides some spatial structure. According to this specification, all the states within a given region are, in a way, considered neighbors that potentially share unobserved similarities. This seems perfectly reasonable given that the regional RE was explicitly built on prior knowledge of distinctive regional voting patterns (see Park, Gelman, and Bafumi 2004, 377). Conditional on the regional RE, however, the state-level RE is assumed independent within and across regions. To see this more clearly, we write out the state-level model in equation (3) to explicitly include an error-term, $v_{j}$

$$
\begin{aligned}
\alpha_{j}^{\text {state }} & =\alpha_{m[j]}^{\text {region }}+\beta^{\text {prev }} \operatorname{prev}_{j}+v_{j}, \\
v_{j} & \sim \mathrm{N}\left(0, \sigma_{v}^{2}\right) .
\end{aligned}
$$

Specifying an adjacency matrix for the U.S. states along the lines of our previous application allows us to directly check the assumption of spatially independent errors. ${ }^{21}$ Results of our replication of Park et al.'s original model including the Moran test for remaining spatial autocorrelation in $v$ are given in Table $4 .^{22}$ Indeed, the Moran statistic indicates moderate but significant spatial autocorrelation in $v(I=0.11)$. Thus, the original model of Park et al. is, though to a modest extent, spatially misspecified in that the regional RE cannot fully account for unobserved similarities among contiguous states. Put differently, there seems some spatial information left to be utilized in the estimation problem. To do so, we replace the regional RE $\alpha_{m[j]}^{\text {region }}$ in equation (12) with a constant term $\alpha^{0}$, and add a CAR-structured RE $\phi_{j}$ instead

$$
\alpha_{j}^{\text {state }}=\alpha^{0}+\beta^{\text {prev }} \operatorname{prev}_{j}+v_{j}+\phi_{j} .
$$

At the same time, we respecify the adjacency matrix that determines $\phi_{j}$ in equation (7) in order not to lose valuable prior information about regional differences in voting patterns and political preferences. In particular, we restrict our neighborhood definition to inner-regional contiguities so that states are considered as neighbors only to the extent that they share a common border or vertex and belong to the same region. ${ }^{23}$ As indicated by a Moran value near zero in Table 4 , the inclusion of $\phi_{j}$ has soaked up the remaining spatial autocorrelation in $v_{j}$. This does not, however, lead to an appreciable improvement of the

\begin{tabular}{|c|c|c|c|c|c|c|c|}
\hline \multicolumn{2}{|c|}{ Mean absolute error } & \multicolumn{2}{|c|}{ Mean width of $90 \%$ CI } & \multicolumn{2}{|c|}{ Coverage probabilities } & \multicolumn{2}{|c|}{ Moran's I } \\
\hline Original & Alternative & Original & Alternative & Original & Alternative & Original & Alternati \\
\hline 0.045 & 0.044 & 0.170 & 0.174 & .922 & .882 & $0.107^{*}$ & 0.001 \\
\hline
\end{tabular}

Table 4 Validation results for the original model of Park, Gelman, and Bafumi (2004), and an alternative model with CAR-structured RE added

${ }^{*} p<.10$ (for Moran values).

\footnotetext{
${ }^{21}$ Accordingly, each state has 4.3 neighbors on average, ranging from 0 for Alaska and Hawaii to 8 for Montana and Tennessee.

${ }^{22}$ Note that Park, Gelman, and Bafumi $(2004,382)$ report an MAE of 4 , not $4.5 \%$. This discrepancy potentially relates to the fact that we did not achieve full convergence with the 5000 iterations suggested by the authors (379). Instead, we ran 10,000 iterations. Detailed results on the parameter estimates are given in the Appendix.

${ }^{23}$ This reduces the average number of neighbors to 3.3. Besides Alaska and Hawaii, Washington, DC has no neighbors according to this definition since Park et al. consider District of Columbia as a separate region. Therefore, $\phi_{j}$ has been set to 0 for these states.
} 
estimates' precision and efficiency. ${ }^{24}$ Considering auxiliary geographic information, it seems, offers little added value in this particular case where ample information on the constituency populations, previous values of the preferences of interest, and affiliation to politico-cultural regions is available.

\section{Discussion}

In this paper, we proposed a Bayesian hierarchical approach to estimating constituency preferences from sparse survey samples that uses auxiliary geographic information in order to compensate for small numbers of respondents per constituency, incomplete constituency coverage, and lack of information about the constituencies' population characteristics. Our model exploited the fact that political preferences often come along in geographical clusters and incorporated the spatial dependence emanating from these clusterings into the model's covariance structure. As a result, the model shrinked directly observed constituency means in survey responses toward the mean responses in neighboring constituencies and toward the whole sample mean. That way, the model borrowed strength both locally and globally to counterbalance small constituency-level samples. Additionally, we employed log inverse constituency area as a proxy of urbanization to account for eventual disruptions of otherwise smooth spatial preference distributions.

We used this model to obtain district estimates of party vote shares at the 2009 German Bundestag election from national postelection surveys that could be validated using official election results. On average, we had less than 20 survey responses per district available — very little information indeed to estimate the vote shares of five parties. Nevertheless, the mean absolute error of our predictions was only $3.4 \%$, and even with smaller samples per district our estimators' properties were still reasonably satisfactory. Also, our model clearly outperformed direct estimates as well as spatially ignorant model-based alternatives.

Building upon these encouraging results, we locate the most fruitful areas of application in similar problems where the number of target areas is high relative to survey sample sizes, and where sociostructural data that could help inform alternative approaches such as the method of Park, Gelman, and Bafumi (2004) is missing. Single-member electoral districts constitute an obvious case (see Rodden 2010), as their number is typically large (e.g., 299 in Germany, 435 in the United States, 650 in the United Kingdom), and detailed census information is rarely available at this level. The same surely holds at even more disaggregated spatial scales such as counties (e.g., Percival, Johnson, and Neiman 2009) or school districts (e.g., Berkman and Plutzer 2005). In fact, spatial dependence often tends to be stronger and thus more informative for our approach, at lower levels of spatial aggregation (see Openshaw and Taylor 1979).

Although our initial model exclusively relied on minimal geographic information readily available from digitalized constituency maps, we further demonstrated how our approach may be integrated with other state-of-the-art strategies that exploit additional information about the constituencies' population characteristics-specifically, hierarchical estimator with poststratification of Park, Gelman, and Bafumi (2004) - to yield even more accurate estimates and to possibly correct for survey nonresponse.

In essence, the strategy we proposed boils down to the insight that spatial correlation is much more than a nuisance for statistical estimation. Rather, spatial dependence can be exploited to help solve problems typically arising when local survey samples are too small to provide reliable estimates of the quantities of interest. The main issue is then how to represent that spatial dependence in order to use it in the estimation problem. Our approach offers one solution to this issue.

\section{Funding}

Center for Quantitative Methods and Survey Research of the University of Konstanz.

\section{References}

Agnew, John. 2002. The making of political geography. Oxford: Oxford University Press.

\footnotetext{
${ }^{24} \mathrm{We}$ have also fitted a model including both the regional and the CAR-structured RE, but we did not achieve convergence even after 30,000 iterations.
} 
Agresti, Alan, and Brent A. Coull. 1998. Approximate is better than "exact" for interval estimation of binomial proportions. American Statistician 52:119-26.

Ansolabehere, Stephen, James M. Snyder, Jr., and Charles Stewart, III. 2001. Candidate positioning in U.S. House elections. American Journal of Political Science 45:136-59.

Ardoin, Phillip J., and James C. Garand. 2003. Measuring constituency ideology in U.S. house districts: A top-down simulation approach. Journal of Politics 65:1165-89.

Bafumi, Joseph, and Michael C. Herron. 2006. Leapfrog representation and extremism: A study of American voters and their members in congress. American Political Science Review 104:519-42.

Berkman, Michael B., and Eric Plutzer. 2005. Ten thousand democracies Politics and public opinion in America's school districts. Washington, DC: Georgetown University Press.

Berry, William D., Richard C. Fording, Evan J. Ringquist, Russell L. Hanson, and Carl Klarner. 2010. Measuring citizen and government ideology in the American states: A re-appraisal. State Politics and Policy Quarterly 10:117-35.

Besag, Julian, Jeremy York, and Annie Molli. 1991. Bayesian image restoration, with two applications in spatial statistics. Annals of the Institute of Statistical Mathematics 43:1-20.

Brace, Paul, Kellie Sims-Butler, Kevin Arceneaux, and Martin Johnson. 2002. Public opinion in the American states: New perspectives using national survey data. American Journal of Political Science 46:173-89.

Canes-Wrone, Brandice, David W. Brady, and John F. Cogan. 2002. Out of step, out of office: Electoral accountability and house members' voting. American Political Science Review 96:127-40.

Carlin, Bradley P., and Thomas A. Louis. 1996. Bayes and empirical Bayes methods for data analysis. London: Chapman \& Hall.

Christensen, William F., and Lindsay Florence. 2008. Predicting presidential and other multistage election outcomes using state-level pre-election polls. American Statistician 62:1-10.

Clinton, Joshua D. 2006. Representation in congress: Constituents and roll calls in the 106th house. Journal of Politics 68: 397-409.

Cressie, Noel A. C. 1993. Statistics for spatial data. 2nd ed. New York: Wiley.

de Sola Pool, Ithiel, Robert P. Abelson, and Samuel L. Popkin. 1965. Candidates, issues, and strategies. Cambridge: MIT Press.

Elazar, Daniel. 1994. The American mosaic The impact of space, time, and culture on American politics. Washington, DC: Westview Press.

Erikson, Robert S. 1978. Constituency opinion and congressional behavior: A reexamination of the Miller-Stokes representation data. American Journal of Political Science 22:511-35.

Erikson, Robert S., and Gerald C. Wright. 1980. Policy representation of constituency interests. Political Behavior 2:91-06.

Erikson, Robert S., Gerald D. Wright, and John P. McIver. 1993. Statehouse democracy Public opinion and policy in the American states. Cambridge: Cambridge University Press.

Gelman, Andrew. 2007. Struggles with survey weighting and regression modeling. Statistical Science 22:153-64

—.2008. Red state, blue state, rich state, poor state Why Americans vote the way they do. Princeton, NJ: Princeton University Press.

Gelman, Andrew, and Thomas C. Little. 1997. Poststratification into many categories using hierarchical logistic regression. Survey Methodology 23:127-35.

Gibson, James L. 1992. The political consequences of intolerance: Cultural conformity and political freedom. American Political Science Review 86:338-56.

Heppen, John. 2003. Racial and social diversity and U.S. Presidential election regions. Professional Geographer 55:191-205.

Hero, Rodney E. 1998. Faces of inequality Social diversity in American politics. Oxford: Oxford University Press.

Hill, Seth J., Michael C. Herron, and Jeffrey B. Lewis. 2010. Economic crisis, Iraq, and race: A study of the 2008 Presidential election. Election Law Journal 9:41-62.

Huefken, Volker. 2010. Supplementary questionnaire and nonresponse-results from the German ISSP survey. Quality \& Quantity 44:607-22.

Hurley, Patricia A., and Kim Quaile Hill. 2003. Beyond the demand-input model: A theory of representational linkages. Journal of Politics 65:304-26.

Jackson, John E. 1989. An error-in-variables approach to estimating models with small area data. Political Analysis 1:157-80.

Jackson, John E., and David C. King. 1989. Public goods, private interests, and representation. American Political Science Review 83:1143-64.

Johnston, Ron, and Charles Pattie. 2006. Putting voters in their place Geography and elections in Britain. Oxford: Oxford University Press.

Kalt, Joseph P., and Mark A. Zupan. 1984. Capture and ideology in the economic theory of politics. American Economic Review 74:279-300.

Kastellec, Jonathan P., Jeffrey R. Lax, and Justin H. Phillips. 2010. Public opinion and Senate confirmation of Supreme Court nominees. Journal of Politics 72:767-84.

Katz, Jonathan N., and Gary King. 1999. A statistical model for multiparty electoral data. American Political Science Review 93:15-32.

Klingemann, H.-D., and B. Wessels. 2001. The political consequences of Germany's mixed-member system: Personalization at the grass roots? In Mixed-member electoral systems The best of both worlds?, ed. M. S. Shugart and M. Wattenberg, 279-96. Oxford: Oxford University Press.

Krehbiel, Keith. 1993. Constituency characteristics and legislative preferences. Public Choice 76:21-37.

Kuklinski, James H. 1978. Representativeness and elections: A policy analysis. American Political Science Review 72:165-77. 
Lax, Jeffrey R., and Justin H. Phillips. 2009a. Gay rights in the states: Public opinion and policy responsiveness. American Political Science Review 103:367-86.

2009b. How should we estimate public opinion in the states? American Journal of Political Science 53:107-21.

Levitt, Steven D. 1996. How do senators vote? Disentangling the role of voter preferences, party affiliation, and senator ideology. American Economic Review 86:425-41.

Luebke, David Martin, and Sybil Milton. 1994. Locating the victim: An overview of census-taking, tabulation technology, and persecution in Nazi Germany. IEEE Annals of the History of Computing 16:25-39.

Lunn, David J., Andrew Thomas, Nicky Best, and David Spiegelhalter. 2000. WinBUGS - A Bayesian modelling framework: Concepts, structure, and extensibility. Statistics and Computing 10:325-37.

McCrone, Donald J., and James H. Kuklinski. 1979. The delegate theory of representation. American Journal of Political Science 23:278-300.

McCrone, Donald J., and Walter J. Stone. 1986. The structure of constituency representation: On theory and method. Journal of Politics 48:956-75.

McDonagh, Eileen Lorenzi. 1993. Constituency influence on house roll-call votes in the progressive era, 1913-1915. Legislative Studies Quarterly 18:185-210.

Miller, Warren E., and Donald E. Stokes. 1963. Constituency influence in congress. American Political Science Review 57:45-56.

Openshaw, S., and P. Taylor. 1979. A million or so correlation coefficients: Three experiments on the modifiable areal unit problem. In Statistical applications in the spatial sciences, ed. N. Wrigley, 127-44. London: Pion.

Park, David K., Andrew Gelman, and Joseph Bafumi. 2004. Bayesian multilevel estimation with poststratification: State-level estimates from national polls. Political Analysis 12:375-85.

Percival, Garrick L., Martin Johnson, and Max Neiman. 2009. Representation and local policy: Relating county-level public opinion to policy outputs. Political Research Quarterly 62:164-77.

Pfefferman, Danny. 2002. Small area estimation. New developments and directions. International Statistical Review 70:125-43.

Pratesi, Monica, and Nicola Salvati. 2008. Small area estimation: The EBLUP estimator based on spatially correlated random area effects. Statistical Methods and Applications 17:113-41.

Rao, J. N. K. 2005. Small area estimation. New York: Wiley.

Rodden, Jonathan. 2010. The geographic distribution of political preferences. Annual Review of Political Science 13:321-40.

Saalfeld, Thomas. 2008. Germany: Stability and strategy in a mixed-member proportional system. In The politics of electoral systems, ed. Michael Gallagher and Paul Mitchell, 209-30. Oxford: Oxford University Press.

Seidman, David. 1975. Simulation of public opinion: A caveat. Public Opinion Quarterly 39:331-42.

Sullivan, John L., and Daniel Richard Minns. 1976. Ideological distance between candidates: An empirical examination. American Journal of Political Science 20:439-68.

Thomas, Andrew, Nicky Best, Dave Lunn, Richard Arnold, and David Spiegelhalter. 2004. GeoBUGS user manual, version 1.2.

Weber, Ronald E, Anne H. Hopkins, Michael L. Mezey, and Frank J. Munger. 1972. Computer simulation of state electorates. Public Opinion Quarterly 36:549-65.

Wright, Gerald C., Robert S. Erikson, and John P. McIver. 1985. Measuring state partisanship and ideology with survey data. Journal of Politics 47:469-89. 